FLOWERS provide nectar for bees, and in return a bee acts as a kind of flying penis, carrying pollen from one plant to fertilise the ovaries of another. Plants and their pollinators are a classic example of mutualism: they have coevolved through evolutionary time in a reciprocal beneficial relationship. However this superficially harmonious view may conceal the true nature of the interaction. It is perhaps more accurate to think of bees having evolved towards efficient exploitation of plants as a food source, while plants have evolved adaptations to minimise the price they pay for getting bees to transfer pollen. G. $\mathbf{H}$. Pyke (Oecologia 36, 281; 1978) has used this line of argument to develop a new insight into the structure of certain inflorescences and the foraging behaviour of bees. He studied three plant genera Delphinium, Aconitum, and Epilobium, all of which are cross-fertilised by bumblebees (Bombus spp.), and have their flowers arranged in a vertical spike.

Bumblebees feeding on these plants follow a characteristic movement pattern. They almost invariably start at the bottom of an inflorescence, move up the spike, and leave to fly to the next plant before reaching the top. Within the inflorescence, a bee typically moves vertically upwards from one flower to the nearest one above. The flowers of all three plant genera are arranged in a spiral around the vertical spike, so that by moving vertically upwards, a bee misses out some of the flowers. The bees' movement rules are related to nectar availability. Flowers at the bottom of an inflorescence are older, bigger and contain more nectar than those at the top; hence by starting at the bottom the bee visits the most profitable

\section{Coevolution of bees and flowers}

\author{
from John R. Krebs
}

flowers first. As it moves up the inflorescence the bee experiences gradually diminishing returns from the successively smaller flowers, until there comes a point at which more nectar per second could be obtained by travelling to the bottom of the next inflorescence. Pyke's measurements suggested that bees roughly follow this optimal departure rule, and this accounts for the fact that a bee usually leaves before reaching the top of an inflorescence. At first sight one might think that it would pay the bee to visit every single flower on its way up the inflorescence, rather than missing nectar-rich flowers near the bottom. However, the spiral arrangement of flowers reduces the benefit of this movement rule. The extra energetic costs to the bee involved in manoeuvering sideways to follow a spiral path more than offset the benefit of visiting all the flowers. The rule of moving straight upwards gives a higher net energy gain.

Bumblebees seem to have evolved a set of rules for efficient exploitation of nectar, but the plants are equally under an evolutionary pressure to exploit bees, and Pyke suggests that the flower arrangement can be interpreted in this way. The larger, older, nectarrich flowers at the bottom of an inflorescence are female, while the younger flowers at the top are male (the plants are protandrous hermaphrodites, meaning that each flower turns from male to female as it gets

John R. Krebs is in the Edward Grey Institute of Field Ornithology, University of Oxford. older). This arrangement of flowers ensures that a visiting bee arrives at the female flowers near the base having just come from the male flowers near the top of the previous plant. In this way the chance of outcrossing is increased, and it is known that in Delphinium at least outcrossing results in a higher percentage seed set (Price \& Waser Nature 277, 294 ; 1979). The spiral arrangement of flowers around the stem is also important to the plant. A bee docs not usually carry enough pollen to fertilise all the female flowers on a plant, so if it visited every flower it would extract some nectar without providing pollen in return. The plants' evoluitionary answer seems to be to arrange its inflorescence in such a way that an efficient bee actually does better by missing some of the female flowers.

Given the design of plants, bumblebees seem to approximate an optimal set of movement rules for maximising net rate of harvesting nectar. At the same time the plants have evolved a design which makes highly efficient (perhaps optimal, but not enough is known about benefits to plants) use of bees as pollinators. It might appear that the coevolutionary struggle has reached a stable point where becs and plants are doing as well as they can, but there is another aspect to the problem. Different specics of plants (as well as different individuals of the same species) compete to attract pollinators, and a complete account of the coevolution of bees and plants must be able to explain why different plants living in the same area are designed to offer nectar in different ways. The answer to this sort of question may emerge from approaches such as Pyke's. for comparison to determine the changes caused by the adsorbate.

A convenient way to explore the effects of the adsorbate is to calculate the surface density of states with and without the adsorbate and to examine the difference in these two functions $\Delta N(E)$. Since the density-of-states function determines the number of electronic states in each energy interval, surface or adsorbate states show up as peaks in this function in the energy interval corresponding to the binding energy of the state. The difference function $\triangle N(E)$ demonstrates how the states near the surface are affected by the adsorbate.

The site where the hydrogen atom is adsorbed on the $\mathrm{Pd}$ (111) surface isn't known, but three different sites are likely. Louie's calculation models all three sites (A, B and C) and computes the $\triangle N(E)$ function for each case
(Fig. 1). This function can be compared with a difference photoemission spectrum (Demuth Surface Sci. 65, $369 ; 1977)$ to determine which geometry fits the measured results.

One prominent feature common to all the $\triangle N(E)$ curves is the dip near the metal Fermi energy $(0 \mathrm{eV})$. This dip reflects the adsorbate correlated movement of states away from this region to lower energy. The peaks at lower energies arise from the new hydrogen induced states formed from hydrogen $1 \mathrm{~s}$ and Pd d-states. Conparison of the $\triangle N(E)$ spectra rules out site $A$ and favours $B$ and $C$ which differ little in their environment. Louie concludes that the hydrogen atoms prefer a threefold hollow over the top site. This geometry yields an occupied H-Pd bonding surface band centred a: $6.5 \mathrm{eV}$ below the Fermi energy (Fig. 1) in good agreement with experiment and an unoccupied antibonding $\mathrm{H}-\mathrm{Pd}$ surface band at high energies (not shown in Fig. 1). Other surface structure is found and analysed.

The features of the $\mathrm{H}-\mathrm{Pd}$ bonding state can be studied using charge density contour maps. These illustrate the details of the mixing between the hydrogen and $\mathrm{Pd}$ orbitals. Louie has also calculated the total electronic charge distribution and finds that some charge spills out into the vacuum above the surface. The values for the number of valence electrons per surface unit cell are 0.2 in the vacuum above the surface and 9.7,10.1,10.0,10.0 for the first, second, third and fourth layers into the bulk ( 10 is the bulk value). The calculated work function is in excellent agreement with experiment.

Although Louie's calculation only considers a specific adsorbate on a specific face of one transition metal, 